\title{
Key Determinants of Optimal Breastfeeding Practices in Laos*
}

\author{
Sengchanh Kounnavong1, Suzinne Pak-Gorstein ${ }^{2,3}$, Kongsap Akkhavong1, Uma Palaniappan ${ }^{4}$, \\ Viorica Berdaga $^{4}$, Joel Conkle ${ }^{5}$, Jonathan Gorstein ${ }^{3,6}$
}

${ }^{1}$ National Institute of Public Health, Vientiane, Laos; ${ }^{2}$ Department of Pediatrics, University of Washington, Seattle, USA; ${ }^{3}$ Global Center for Integrated Health of Women, Adolescents, and Children, University of Washington, Seattle, USA; ${ }^{4}$ UNICEF-Laos, Vientiane, Laos; ${ }^{5}$ UNICEF-Cambodia, Phnom Penh, Cambodia; ${ }^{6}$ Department of Global Health, University of Washington, Seattle, USA.

Email: gorstein@uw.edu

Received July 6 ${ }^{\text {th }}, 2013$; revised August $6^{\text {th }}, 2013$; accepted August $15^{\text {th }}, 2013$

Copyright (c) 2013 Sengchanh Kounnavong et al. This is an open access article distributed under the Creative Commons Attribution License, which permits unrestricted use, distribution, and reproduction in any medium, provided the original work is properly cited.

\begin{abstract}
This paper provides information on optimal breastfeeding practices in the People's Democratic Republic of Lao from data collected in the 2011 Lao Social Indicator Survey. Results: Early initiation of breastfeeding within the first hour of life occurred among $39.6 \%$ of Laotian infants. After controlling for the effects of wealth, early initiation of breastfeeding was found to be most prevalent among mothers with higher education, those who received antenatal services, and those who delivered in a health facility. Avoidance of prelacteal feeds within the first three days of life occurred among 65\% of Laotian infants, and was highest among ethnic Hmong and Khmer infants, and among those born in public versus private health facilities. Avoidance of prelacteal feeds was lowest after deliveries in which a traditional birth attendant was present. Exclusive breastfeeding through the first 5 months of age was reported among $40.8 \%$ of infants nationally. Ethnicity again played a role with the highest levels of exclusive breastfeeding found to occur among ethnic Khmer infants (69.4\%, OR 2.8, CI: 1.5 - 5.1). Discussion: These results highlight the role that health care workers can have on early breastfeeding practices at the point of both antenatal counseling and in the delivery setting. Strengthening the quality of counseling on infant feeding can have a significant impact on early initiation of breastfeeding. Ethnic differences significantly impact both early and exclusive breastfeeding practices. Conclusion: A complexity of factors at different levels of care impact breastfeeding practices. A synergy of strategic approaches are needed to target antenatal counseling as well as post-delivery practices, all which account for the unique social and cultural attitudes towards infant feeding. Stronger community-based interventions that account for cultural attitudes and practices are most likely to be successful in promoting exclusive and continued breastfeeding practices.
\end{abstract}

Keywords: Breastfeeding; Infant and Child Nutrition; Laos; MYCNSIA; Complimentary Feeding

\section{Introduction}

The early period in life is critical for cognitive, mental, and physical development. Adequate nutrition during this period ensures proper development and plays a significant role in lifelong health outcomes. Inadequate nutrition during pregnancy and the first two years of life can irreversibly limit the ability of a child to achieve his or her full potential. These adverse outcomes, which can begin before birth, may include delayed mental devel-

\footnotetext{
*This paper has been produced with the financial assistance of the European Union and UNICEF. The views expressed herein can in no way be taken to reflect the official opinion of the European Union or UNICEF.
}

opment, reduced mental capacity, reduced school performance and working capacity, and overall increased susceptibility to infections and diseases. An undernourished child has an increased risk of dying from diarrhea, measles, malaria and pneumonia. The first 1000 days of life from conception through the first two years of life is often called the "window of opportunity" because of the profound positive effect optimal nutrition can have on the developing child and due to the fact that damage sustained during this period is often permanent [1].

\section{IYCF and Breastfeeding Indicators}

Programs have been in place to promote and support op- 
timal feeding and nutrition for newborns and in the first two years of life. Infant and young child feeding (IYCF) indicators were established in 2007 by the World Health Organization (WHO) as a means of tracking progress of efforts to encourage and promote appropriate feeding practices. These indicators were developed over a five year period in order to establish simple, valid and reliable metrics to assess the prevalence of breastfeeding and complementary feeding practices in children less than two years of age. Prior to the establishment of standardized IYCF indicators, consensus on appropriate feeding practices for children under two years of age did not exist and it was difficult to track progress and changes in nutrition resulting from different interventions.

Both exclusive and early initiation of breastfeeding promotes the health of an infant, and these are recognized to be some of the most cost-effective interventions to reduce the overall burden of disease in a population [2]. During the first few months of life, breastmilk provides all of the nutrients necessary for development and has many other important attributes. Infants who are exclusively breastfed to six months of age have lower mortality from childhood illnesses such as pneumonia and diarrhea, and rebound quicker from periods of sickness [3-6]. Colostrum, the first milk expressed after delivery, is rich in nutrients and antibodies. The high concentration of nutrients and antibodies found in colostrum enables delivery in a low-volume form, which is essential because of the small size of a newborn's digestive system. Early initiation of breastfeeding, which the WHO defines as within one hour of birth, guarantees that newborns receive this nutrient-rich liquid [7]. Adherence to this practice ensures that maternal antibodies are passed to the infant protecting the newborn from illnesses and reducing infant mortality. Early initiation of breastfeeding has also been linked to longer duration of breastfeeding. The definitions for the IYCF indicators related to breastfeeding and complementary feeding are listed in Table $\mathbf{1 .}$

While generating robust estimates and tracking trends on the prevalence of different feeding practices is important, it is critical to understand the main factors associated with optimal adherence to breastfeeding guidelines. Identification of the key determinants of breastfeeding is imperative to design messages and target resources for the effective implementation of IYCF programs. Multicountry comparisons with other Asian countries have shown that rates vary considerably by country, and that factors associated with poor feeding practices are not always consistent across countries [8].

Inter-country variations in breastfeeding determinants indicates a need to assess the local factors which drive feeding practices in order to tailor programs for improving infant and young child nutrition. Within country variation of IYCF practices are also likely which high-
Table 1. IYCF breastfeeding indicators.

\section{CORE IYCF BREASTFEEDING INDICATORS}

\section{Early initiation of breastfeeding}

Proportion of children born in the last 24 months who were put to the breast within one hour of birth.

Exclusive breastfeeding under 6 months

Proportion of infants $0-5$ months of age who are fed exclusively with breast milk.

Continued breastfeeding at 1 year

Proportion of children 12 - 15 months of age who are fed breast milk.

\section{OPTIONAL IYCF BREASTFEEDING INDICATORS}

\section{Children ever breastfed}

Proportion of children born in the last 24 months who were ever breastfed.

Predominant breastfeeding under 6 months

Proportion of infants 0 - 5 months of age who are predominately breastfed.

Continued breastfeeding at 2 years

Proportion of children 20 - 23 months of age who are fed breast milk.

Age-appropriate breastfeeding

Proportion of children 0 - 23 months who are appropriately breastfed.

Bottle feeding

Proportion of children 0 - 23 months of age who are fed with a bottle.

lights the importance of analyzing patterns and associated risk factors within small geographic areas of a country. Procuring local evidence of the determinants of IYCF practices are an important step in defining effective programs.

Recognizing the reasons why women do not or are not able to breastfeed often go beyond their "knowledge" of its importance and may be related to social norms, traditions, the enabling environment and community support mechanisms. While challenges and obstacles to adopting IYCF recommendations have been identified for other countries [9], analysis needs to be tailored to individual country settings and contexts. This paper aims to identify some of the key characteristics of mothers who practice optimal breastfeeding in Laos. The data which inform this analysis were collected as part of a large national survey on health and nutrition.

\section{Methods}

\subsection{MYCNSIA and LSIS Survey and Study Sites}

The European Union (EU)-UNICEF joint action is supporting the Maternal and Young Child Nutrition Security in Asia (MYCNSIA) Project in five countries: Bangladesh, Indonesia, Lao PDR, Nepal, and the Philippines. The four-year MYCNSIA was initiated in Lao PDR in 2011 and aims to strengthen the National Plan of Action for Nutrition (NPAN) to improve child survival, growth and development through nutrition security interventions. A major focus of the MYCNSIA Project is to develop 
and implement innovative interventions to improve maternal and child nutrition during the greatest period of vulnerability; namely, the "first 1000 days" of life from conception, through the first two years of a child's life.

Prior to the introduction of key interventions, baseline data were collected to characterize the study population. The baseline survey was actually derived from two different sources, the national Lao Social Indicator Survey (LSIS) and a supplementary MYCNSIA survey conducted in the three Provinces. The field work for both surveys was carried out between October 2011 and February 2012. The LSIS was implemented by the Ministry of Health and the Lao Statistical Bureau with support from UNICEF, UNFPA, and other donors. The survey was based on the design of the Multiple Indicator Cluster Survey (MICS), the Demographic and Health Survey (DHS) and the Lao Reproductive Health Survey (LRHS).

\subsection{Sample Design}

The LSIS sample frame was designed to derive statistically robust and independent estimates for each province in the country. Census cluster areas within each province were selected systematically using a square root allocation method. Cluster numbers were then adjusted to attain a minimum of 50 clusters in each province and a maximum of 75 in the largest provinces. Within each cluster, households were listed and a systematic sample of 20 households selected. Statistical weights were developed in order to adjust for household size, cluster size, and the total population of the individual provinces, which assured that aggregate estimates were representative of the population at the provincial level.

\subsection{Field Team Training}

Details on the core LSIS field team management are available in the LSIS Report [10]. Each team was composed of four individuals with specific roles: supervisor, team leader, interviewer, and laboratory technician. Supervisors, teams leaders and interviewers all conducted face-to-face interviews. Enumerator training was carried out and scores from a pre- and post-test verified that key interviewing protocols and anthropometric assessments were correctly performed by field workers using standardized WHO procedures.

\subsection{Statistical Analysis}

Data entry was completed in March 2012 and analyzed using Statistical Package for Social Sciences (SPSS) version 19. Frequencies were run to describe the basic characteristics of each variable of interest and to ensure that data were collected from each individual. Missing data were coded as missing and excluded from analysis.

Only data from children that had merged information with mother data were used for this analysis in order to capture the maternal level covariates. Comparative analyses of those cases that matched with those that did not match revealed no significant differences in any of the major outcome covariates such as child growth and maternal age. The range of cases that did not match ranged from $4 \%$ to $6 \%$ for each province. Also, it is for this reason that the estimates of major indicators differed from the national estimates reported, although the differences were statistically insignificant. In addition, only children who were born in the last 2 years were included in this analysis since questions on initiation of breastfeeding at birth were only asked of those mothers with children under 2 years of age due to concerns about recall.

Key outcome variables as described above were stratified by multiple covariates to capture significant differences between groups with special attention to identify inequalities between sub-groups of the population. Frequencies for each program coverage and outcome variable were stratified by sex, age, province, residence, rural area, maternal education, wealth index quintile, and ethnicity, language, and religion. Bivariate analyses including Chi square tests were performed to determine statistical significance of differences for outcomes. Simple binary logistic regressions were performed in order to calculate crude odds ratios and their p-values. Multivariate logistic regression models were also developed to calculate adjusted odds ratios, controlling for key factors to better identify the main determinants of optimal breastfeeding practices.

\section{Results}

\subsection{Demographic Characteristics}

Table 2 shows the individual, household and community level characteristics of the 4322 children aged $6-23$ months enumerated as part of the LSIS. Of these children, $26.9 \%$ were under 6 months of age, $25.5 \%$ were between 6 and 11 months of age and $47.6 \%$ between 12 and 23 months of age. Over three-quarter of the children (77.3\%) reside in rural areas. The majority of the mothers were either uneducated or had just received some primary level education (69.7\%). The households were primarily from the majority Laotian ethnic group, with half of the population practiced Buddhism, while the other half Animism. It is noteworthy that the households with children under two years of age included in this analysis from the LSIS were somewhat less affluent than the general population, as evidenced by the higher percentage of children in the poorest wealth quintile as compared to other stratum of economic status.

\subsection{Early Initiation of Breastfeeding}

Table 3 shows the proportion of mothers who breastfed 
Table 2. Basic demographic profile of children age 0 - 23 months of age-Lao Social Indicator Survey (LSIS) 2011 .

\begin{tabular}{|c|c|c|}
\hline Variable & $N$ & $\%$ \\
\hline \multicolumn{3}{|l|}{ Age group (months) } \\
\hline $0-5$ & 1164 & 26.9 \\
\hline $6-11$ & 1103 & 25.5 \\
\hline $12-23$ & 2055 & 47.6 \\
\hline \multicolumn{3}{|l|}{ Sex of infant } \\
\hline Male & 2182 & 50.5 \\
\hline Female & 2140 & 49.5 \\
\hline \multicolumn{3}{|l|}{ Mother's age (years) } \\
\hline $15-19$ & 518 & 12.0 \\
\hline $20-24$ & 1326 & 30.8 \\
\hline $25-29$ & 1175 & 27.2 \\
\hline $30-34$ & 673 & 15.6 \\
\hline $35+$ & 630 & 14.6 \\
\hline \multicolumn{3}{|l|}{ Mother's education } \\
\hline None & 1259 & 29.1 \\
\hline Primary & 1755 & 40.6 \\
\hline Secondary and above & 1308 & 30.3 \\
\hline \multicolumn{3}{|l|}{ Ethnicity } \\
\hline Lao - Tai & 2074 & 48.0 \\
\hline Mon - Khmer & 502 & 11.6 \\
\hline Hmong & 544 & 12.6 \\
\hline Other & 1197 & 27.7 \\
\hline \multicolumn{3}{|l|}{ Religion } \\
\hline Buddhist & 2473 & 57.3 \\
\hline Animist & 1783 & 41.3 \\
\hline Other & 59 & 1.4 \\
\hline \multicolumn{3}{|l|}{ Household wealth index } \\
\hline Poorest & 1163 & 26.9 \\
\hline Second quintile & 922 & 21.3 \\
\hline Third quntile & 813 & 18.8 \\
\hline Fourth quintile & 728 & 16.8 \\
\hline Wealthiest & 696 & 16.1 \\
\hline \multicolumn{3}{|l|}{ Residence } \\
\hline Urban & 983 & 22.7 \\
\hline Rural & 3339 & 77.3 \\
\hline \multicolumn{3}{|l|}{ Region } \\
\hline North & 1371 & 31.7 \\
\hline Central & 2012 & 46.6 \\
\hline South & 939 & 21.7 \\
\hline
\end{tabular}

${ }^{\mathrm{a}}$ Weighted by sample cluster size.

their last borne child under two years of age within the first hour after birth, along with odds ratio for key predictors.

The national prevalence of early breastfeeding initiation within the first hour of life was 39.6\%. Low levels of early breastfeeding initiation are seen across all ethnic groups. Higher levels of early initiation of breastfeeding were noted among mothers who received antenatal care $(\mathrm{OR}=2.0,95 \%$ CI: $1.7-2.2)$, delivered with the assistance of a skilled attendant (OR = 3.8, 95\% CI: 2.7 - 5.3), delivered at a public or private facility ( $\mathrm{OR}=1.9,95 \%$ C.I: 1.0 - 3.5), and who possessed higher education.

Gender and current age of the child were also analyzed, but not found to have a statistically significant bearing on early breastfeeding practices (data not shown).

The proportion of infants breastfed within the first 24 hours of life was also collected and analyzed. The survey revealed $72.9 \%$ of Laotian mothers reporting having breastfed their infant within the first day after delivery which compares well with the $70.5 \%$ figure reported from the national report that included all children regardless of their data matching with mother-level questions.

The proportion of children who had been breastfed within the first 24 hours of life was expectedly higher than the proportion breastfed within the first hour. The influence of predictors for early breastfeeding in the first day was similar so that, for example, the presence of a professional skilled attendant at delivery possessing a strong association with nursing within the first 24 hours of life (80.4\% compared to $45.1 \%$ for infants born with no one assisting, $(\mathrm{OR}=10.4,95 \% \mathrm{CI}$ : $8.0-13.5, \mathrm{p}<$ 0.001).

\subsection{Prelacteal Feeds}

Prelacteal feeds are those foods and fluids fed to newborns before breastfeeding is established usually on the first day of life. Javascript: newshowcontent ("active", "references"); The World Health Organization recommends avoiding any prelacteal feeds, as other liquids are suboptimal in comparison to breastmilk and will quickly fill a newborn's small stomach, thus interfering with breastfeeding and potentially exposing them to environmental pathogens. Although this is not an IYCF indicator the results revealed important findings for breastfeeding practices in Laos and are reported here.

Overall, as seen in Table 4, 65.0\% of all children in Laos avoided prelacteal fluids during their first 3 days of life, while the inverse, or $35.0 \%$ of children were given some kind of prelacteal fluid. Mothers with higher levels of education avoided prelacteal feeding at somewhat higher proportions than mothers with lower or no education, but this only reached statistical significance when controlling for other covariates (adjusted OR $=1.5$ ). Prelacteal feeds were composed of primarily plain water (80\%) or other milks (data not shown).

Receipt of antenatal care did not significantly alter rates of prelacteal feeds. However, when a traditional birth attendant (TBA) was present at birth, the infant was much more likely to be given a prelacteal feed as compared to a professional skilled attendant or even when assisted by an untrained family or friend, which shows the strong role of social norms in these practices. In addition, infants were more likely to avoid being given prelacteals in their first 3 days of life if they were born in a public health facility as compared to if they were born at home. Children born in private health facilities were the least likely to avoid being given prelacteals, which is likely related to the use of breastmilk substitutes. Finally, ethnic heritage of the family was the largest factor associated with avoidance of prelacteals. Prelacteal feeding 
Table 3. Predictors of early initiation of breastfeeding (fed breastmilk within first hour of life)—Lao Social Indicator Survey (LSIS) 2011 ${ }^{\mathrm{a}}$.

\begin{tabular}{|c|c|c|c|c|}
\hline Variable & $n$ & $(\%)$ & Crude OR $(95 \% \text { CI })^{\mathrm{b}}$ & Adjusted OR (95\% CI) \\
\hline \multicolumn{5}{|l|}{ Mother's age (yrs) } \\
\hline $15-19$ & 518 & 36.7 & & \\
\hline $20-24$ & 1326 & 40.9 & $1.2(1.0-1.5)$ & $1.2(0.9-1.5)$ \\
\hline $25-29$ & 1175 & 42.2 & $1.3(1.0-1.5)$ & $1.1(0.9-1.4)$ \\
\hline $30-34$ & 673 & 41.6 & $1.2(1.0-1.5)$ & $1.0(0.8-1.3)$ \\
\hline $35+$ & 630 & 32.0 & $0.8(0.6-1.0)^{*}$ & $0.8(0.6-1.1)$ \\
\hline \multicolumn{5}{|l|}{ Mother's education } \\
\hline None & 1259 & 31.6 & & \\
\hline Primary & 1755 & 37.9 & $1.3(1.1-1.5)^{* * * *}$ & $1.3(1.1-1.6)^{* *}$ \\
\hline Secondary and above & 1308 & 49.5 & $2.1(1.8-2.5)^{* * *}$ & $1.7(1.4-2.1)^{* * * *}$ \\
\hline \multicolumn{5}{|l|}{ Received antenatal care } \\
\hline No & 1805 & 31.2 & & \\
\hline Yes & 2437 & 47.1 & $2.0(1.7-2.2)^{* * *}$ & $1.6(1.3-1.9)^{* * *}$ \\
\hline \multicolumn{5}{|l|}{ Place of delivery } \\
\hline Home & 2507 & 34.3 & & \\
\hline Public facility & 1578 & 49.9 & $1.9(1.7-2.2)^{* * *}$ & $1.5(1.1-2.1)^{*}$ \\
\hline Private facility & 42 & 50.1 & $1.9(1.0-3.5)^{*}$ & $1.5(0.7-3.0)$ \\
\hline \multicolumn{5}{|l|}{ Assistance at delivery } \\
\hline No one & 341 & 20.5 & & \\
\hline Family/Friend & 1323 & 38.5 & $2.4(1.7-3.4)^{* * *}$ & $1.6(1.0-2.5)^{*}$ \\
\hline Trad'l birth att & 497 & 32.6 & $1.9(1.3-2.7)^{* *}$ & $1.0(0.6-1.7)$ \\
\hline Prof skilled att & 1792 & 49.3 & $3.8(2.7-5.3)^{* * * *}$ & $1.2(0.7-2.1)$ \\
\hline \multicolumn{5}{|l|}{ Ethnicity } \\
\hline Lao - Tai & 2074 & 43.2 & & \\
\hline Mon - Khmer & 502 & 33.9 & $0.7(0.5-0.8)^{* * *}$ & $0.5(0.4-0.8)^{* *}$ \\
\hline Hmong & 544 & 34.5 & $0.7(0.6-0.8)^{* * *}$ & $0.7(0.5-1.0)$ \\
\hline Other & 1197 & 37.9 & $0.8(0.7-0.9)^{* *}$ & $0.9(0.7-1.1)$ \\
\hline \multicolumn{5}{|l|}{ Religion } \\
\hline Buddhist & 2473 & 42.2 & & \\
\hline Animist & 1783 & 35.8 & $0.8(0.7-0.9)^{* * * *}$ & $1.4(1.1-1.9)^{* *}$ \\
\hline Other & 59 & 40.3 & $0.9(0.5-1.6)$ & $1.8(1.0-3.4)^{*}$ \\
\hline \multicolumn{5}{|l|}{ Household wealth index } \\
\hline Poorest & 1163 & 32.3 & & \\
\hline $2^{\text {nd }}$ quintile & 922 & 36.0 & $1.2(1.0-1.4)$ & $0.9(0.7-1.1)$ \\
\hline $3^{\text {rd }}$ quintile & 813 & 39.4 & $1.4(1.1-1.6)^{* *}$ & $1.0(0.8-1.3)$ \\
\hline $4^{\text {th }}$ quintile & 728 & 46.0 & $1.8(1.4-2.1)^{* * *}$ & $1.2(0.9-1.6)$ \\
\hline Wealthiest & 696 & 49.9 & $2.1(1.7-2.4)^{* * *}$ & $1.2(0.9-1.6)$ \\
\hline \multicolumn{5}{|l|}{ Residence } \\
\hline Rural & 3339 & 37.5 & & \\
\hline Urban & 983 & 46.5 & $1.4(1.2-1.6)^{* * *}$ & $0.9(0.8-1.1)$ \\
\hline \multicolumn{5}{|l|}{ Region } \\
\hline North & 1371 & 43.2 & & \\
\hline Central & 2012 & 36.5 & $0.7(0.6-0.8)^{* * *}$ & $0.5(0.4-0.6)^{* * *}$ \\
\hline South & 939 & 40.8 & $0.9(0.7-1.0)$ & $0.8(0.7-1.0)$ \\
\hline Total & 4322 & 39.6 & & \\
\hline
\end{tabular}


Table 4. Predictors for avoiding prelacteal feeds in the first three days of life-Lao Social Indicator Survey (LSIS) $2011{ }^{\mathrm{a}}$.

\begin{tabular}{|c|c|c|c|c|}
\hline Variable & $n$ & $(\%)$ & OR 95\% $\mathrm{CI}^{\mathrm{b}}$ & Adjusted OR (95\% CI) \\
\hline \multicolumn{5}{|l|}{ Mother’s age(years) } \\
\hline $15-19$ & 518 & 66.3 & & \\
\hline $20-24$ & 1326 & 68.7 & $1.1(0.9-1.4)$ & $1.2(1.0-1.6)$ \\
\hline $25-29$ & 1175 & 65.0 & $0.9(0.8-1.2)$ & $1.1(0.9-1.5)$ \\
\hline $30-34$ & 673 & 63.5 & $0.9(0.7-1.1)$ & $1.2(0.9-1.7)$ \\
\hline $35+$ & 630 & 57.4 & $0.7(0.5-0.9)$ & $0.9(0.7-1.3)$ \\
\hline \multicolumn{5}{|l|}{ Mother's education } \\
\hline None & 1259 & 65.1 & & \\
\hline Primary & 1755 & 64.0 & $0.9(0.8-1.1)$ & $1.2(1.0-1.6)^{*}$ \\
\hline Secondaryand above & 1308 & 66.5 & $1.1(0.9-1.3)$ & $1.5(1.2-2.0)^{* *}$ \\
\hline \multicolumn{5}{|l|}{ Received antenatal care } \\
\hline No & 1805 & 65.4 & & \\
\hline Yes & 2437 & 64.5 & $1.0(0.9-1.2)$ & $1.2(1.0-1.5)$ \\
\hline \multicolumn{5}{|l|}{ Place of delivery } \\
\hline Home & 2507 & 63.1 & & \\
\hline Public facility & 1578 & 67.3 & $1.2(1.0-1.4)^{* *}$ & $2.1(1.5-2.9)^{* * *}$ \\
\hline Privatefacility & 42 & 51.5 & $0.6(0.3-1.1)$ & $1.1(0.5-2.3)$ \\
\hline \multicolumn{5}{|l|}{ Assistance at delivery } \\
\hline No one & 341 & 70.6 & & \\
\hline Family/Friend & 1323 & 72.7 & $1.1(0.7-1.6)$ & $1.2(0.8-1.9)$ \\
\hline Trad'l birth att & 497 & 45.0 & $0.3(0.2-0.5)^{* * *}$ & $0.6(0.4-1.0)^{*}$ \\
\hline Prof skilled att & 1792 & 65.8 & $0.8(0.5-1.2)$ & $0.7(0.4-1.2)$ \\
\hline \multicolumn{5}{|l|}{ Ethnicity } \\
\hline Lao - Tai & 2074 & 57.0 & & \\
\hline Mon - Khmer & 502 & 81.5 & $3.1(2.4-4.0)^{* * *}$ & $1.1(0.7-1.6)$ \\
\hline Hmong & 544 & 86.4 & $4.5(3.4-5.8)^{* * *}$ & $3.2(2.2-4.9)^{* * *}$ \\
\hline Other & 1197 & 62.0 & $1.2(1.0-1.4)^{*}$ & $1.0(0.8-1.2)$ \\
\hline \multicolumn{5}{|l|}{ Religion } \\
\hline Buddhist & 2473 & 57.7 & & \\
\hline Animist & 1783 & 75.1 & $2.1(1.8-2.4)^{* * *}$ & $1.4(1.1-1.9)^{*}$ \\
\hline Other & 59 & 62.9 & $1.2(0.7-2.1)$ & $0.6(0.6-2.1)$ \\
\hline \multicolumn{5}{|l|}{ Household wealth index } \\
\hline Poorest & 1163 & 69.3 & & \\
\hline $2^{\text {nd }}$ quintile & 922 & 63.8 & $0.8(0.6-0.9)^{*}$ & $0.8(0.6-1.0)$ \\
\hline $3^{\text {rd }}$ quintile & 813 & 66.7 & $0.9(0.7-1.1)$ & $1.0(0.8-1.3)$ \\
\hline $4^{\text {th }}$ quintile & 728 & 61.1 & $0.7(0.6-0.8)^{* *}$ & $0.8(0.6-1.1)$ \\
\hline Wealthiest & 696 & 61.5 & $0.7(0.6-0.9)^{* *}$ & $0.8(0.5-1.1)$ \\
\hline \multicolumn{5}{|l|}{ Residence } \\
\hline Rural & 3339 & 65.2 & & \\
\hline Urban & 983 & 64.5 & $1.0(0.8-1.1)$ & $1.1(0.9-1.4)$ \\
\hline \multicolumn{5}{|l|}{ Region } \\
\hline North & 1371 & 85.7 & & \\
\hline Central & 2012 & 54.6 & $0.2(0.1-0.2)^{* * *}$ & $0.2(0.2-0.3)^{* * *}$ \\
\hline South & 939 & 56.7 & $0.2(0.2-0.3)^{* * * *}$ & $0.3(0.3-0.4)^{* * *}$ \\
\hline Total & 4322 & 65.0 & & \\
\hline
\end{tabular}

does not appear to be the social norm for the Hmong, with $85 \%$ of children in this ethnic group avoiding prelacteals.

\subsection{Exclusive Breastfeeding}

Children who are exclusively breastfed receive no foods or other liquids apart from breast milk, although oral rehydration solution (ORS), vitamins, minerals and me- dicines in drops and syrups are allowed.

Overall, $40.8 \%$ of children 0 - 5 months of age were being exclusively breastfed in this survey (Table 5). Children who are not exclusively breastfed are receiving water, early complementary feeding, and breastmilk substitutes. Neither the gender of the child nor the age of the mother was associated with significant differences in the probability of exclusive breastfeeding for children under 
6 months of age. The region of the country emerged as one of the most important determinants of exclusive breastfeeding, where mothers in the central and southern parts of the country were about half as likely to practice exclusive breastfeeding than in the north. Region of the country is closely linked to ethnicity; infants from MonKhmer and Hmong families were more likely to be breastfed then their Laotian counterparts. Religion of the household was seen as being more important in exclusive breastfeeding than observed for early initiation of breastfeeding, although did not reach statistical significance.

\subsection{Continued Breastfeeding}

Continued breastfeeding is encouraged beyond six months of age as it provides half or more of the daily caloric needs for children between 6 - 11 months of age, and about a third of the dietary energy requirements for children in the second year of life.

While the gender of the child was not associated with a higher tendency for continued breastfeeding, mothers aged 35 years or older were more likely to report continuing to breastfeed their children at one year of age than younger mothers (Table 6). Mothers with low

Table 5. Predictors of Exclusive Breastfeeding among Laotian children 0 - 5 months of age-Lao Social Indicator Survey (LSIS) 2011

\begin{tabular}{|c|c|c|c|c|}
\hline Variable & $n$ & (\%) & OR 95\% $\mathrm{CI}^{\mathrm{b}}$ & Adjusted OR $(95 \% \text { CI })^{b}$ \\
\hline \multicolumn{5}{|l|}{ Sex of child } \\
\hline Male & 566 & 39.1 & & \\
\hline Female & 598 & 42.4 & $1.1(0.9-1.4)$ & $1.1(0.7-1.4)$ \\
\hline \multicolumn{5}{|l|}{ Mother's age(years) } \\
\hline $15-19$ & 194 & 43.1 & & \\
\hline $20-24$ & 391 & 42.7 & $1.0(0.7-1.4)$ & $1.0(0.7-1.5)$ \\
\hline $25-29$ & 273 & 41.7 & $0.9(0.6-1.4)$ & $1.0(0.7-1.5)$ \\
\hline $30-34$ & 163 & 38.4 & $0.8(0.5-1.3)$ & $0.9(0.6-1.5)$ \\
\hline $35+$ & 142 & 33.5 & $0.7(0.4-1.0)$ & $0.7(0.5-1.2)$ \\
\hline \multicolumn{5}{|l|}{ Mother's education } \\
\hline None & 338 & 45.4 & & \\
\hline Primary & 450 & 35.7 & $0.7(0.5-0.9)^{* *}$ & $0.8(0.6-1.2)$ \\
\hline Secondaryand above & 375 & 42.9 & $0.9(0.9-1.2)$ & $1.2(0.8-1.9)$ \\
\hline \multicolumn{5}{|l|}{ Ethnicity } \\
\hline Lao - Tai & 561 & 33.1 & & \\
\hline Mon - Khmer & 120 & 57.1 & $2.7(1.8-4.0)^{* * * *}$ & $1.3(0.7-2.5)$ \\
\hline Hmong & 159 & 69.4 & $4.6(3.1-6.8)^{* * *}$ & $2.8(1.5-5.1)^{* *}$ \\
\hline Other & 323 & 34.2 & $1.0(0.8-1.4)$ & $0.8(0.5-1.2)$ \\
\hline \multicolumn{5}{|l|}{ Religion } \\
\hline Buddhist & 673 & 32.9 & & \\
\hline Animist & 473 & 52.0 & $2.2(1.7-2.8)^{* * *}$ & $1.3(0.8-2.0)$ \\
\hline Other & 16 & 45.7 & $1.7(0.6-4.6)$ & $1.3(0.4-4.0)$ \\
\hline \multicolumn{5}{|l|}{ Household wealth index } \\
\hline Poorest & 281 & 46.8 & & \\
\hline $2^{\text {nd }}$ quintile & 272 & 39.6 & $0.7(0.5-1.0)$ & $0.9(0.6-1.3)$ \\
\hline $3^{\text {rd }}$ quintile & 235 & 42.5 & $0.8(0.6-1.2)$ & $1.1(0.7-1.7)$ \\
\hline $4^{\text {th }}$ quintile & 194 & 37.8 & $0.7(0.5-1.0)^{*}$ & $1.1(0.6-1.8)$ \\
\hline Wealthiest & 182 & 34.6 & $0.6(0.4-1.0)^{*}$ & $0.9(0.5-1.7)$ \\
\hline \multicolumn{5}{|l|}{ Residence } \\
\hline Rural & 926 & 41.5 & & \\
\hline Urban & 238 & 38.4 & $0.9(0.6-1.2)$ & $1.0(0.7-1.4)$ \\
\hline \multicolumn{5}{|l|}{ Region } \\
\hline North & 352 & 60.7 & & \\
\hline Central & 546 & 33.2 & $0.3(0.2-0.4)^{* * *}$ & $0.4(0.3-0.5)^{* * *}$ \\
\hline South & 265 & 30.1 & $0.3(0.2-0.4)^{* * *}$ & $0.4(0.3-0.7)^{* * *}$ \\
\hline Total & 1164 & 40.8 & & \\
\hline
\end{tabular}

${ }^{\mathrm{a}}$ Child born within two years preceding the survey; ${ }^{\mathrm{b}^{*}} p<0.05,{ }^{* *} p<0.01,{ }^{* * *} p<0.001$. 
Table 6. Predictors of Continued Breastfeeding among Laotian children at one year of age-Lao Social Indicator Survey (LSIS) 2011

\begin{tabular}{|c|c|c|c|c|}
\hline Variable & $n$ & $(\%)$ & OR $95 \% \mathrm{CI}^{\mathrm{b}}$ & Adjusted OR $(95 \% \mathrm{CI})^{\mathrm{b}}$ \\
\hline \multicolumn{5}{|l|}{ Sex of child } \\
\hline Male & 364 & 76.1 & & \\
\hline Female & 361 & 74.6 & $0.9(0.7-1.3)$ & $0.9(0.7-1.3)$ \\
\hline \multicolumn{5}{|l|}{ Mother's age(years) } \\
\hline $15-19$ & 63 & 68.1 & & \\
\hline $20-24$ & 231 & 75.2 & $1.3(0.7-2.4)$ & $1.4(0.7-2.8)$ \\
\hline $25-29$ & 206 & 69.8 & $1.0(0.5-1.8)$ & $1.2(0.6-2.4)$ \\
\hline $30-34$ & 114 & 81.0 & $2.0(0.9-4.2)$ & $2.5(1.1-5.6)^{*}$ \\
\hline $35+$ & 112 & 84.3 & $2.9(1.3-6.5)^{* *}$ & $3.3(1.4-7.9)^{* *}$ \\
\hline \multicolumn{5}{|l|}{ Mother's education } \\
\hline None & 221 & 87.8 & & \\
\hline Primary & 293 & 75.6 & $0.4(0.2-0.6)^{* * *}$ & $0.7(0.4-1.3)$ \\
\hline Secondary and above & 212 & 62.2 & $0.2(0.1-0.4)^{* * *}$ & $0.7(0.4-1.3)$ \\
\hline \multicolumn{5}{|l|}{ Ethnicity } \\
\hline Lao - Tai & 327 & 65.6 & & \\
\hline Mon - Khmer & 90 & 86.3 & $3.0(1.6-5.8)^{* *}$ & $1.2(0.5-3.2)$ \\
\hline Hmong & 99 & 89.0 & $3.9(2.0-7.7)^{* * *}$ & $1.6(0.6-4.4)$ \\
\hline Other & 207 & 79.2 & $2.2(1.4-3.3)^{* * *}$ & $1.0(0.5-1.8)$ \\
\hline \multicolumn{5}{|l|}{ Religion } \\
\hline Buddhist & 401 & 66.3 & & \\
\hline Animist & 315 & 87.2 & $3.3(2.2-4.9)^{* * *}$ & $1.4(0.7-3.0)$ \\
\hline Other & 8 & 61.6 & $0.7(0.2-3.0)$ & $0.5(0.1-2.5)$ \\
\hline \multicolumn{5}{|l|}{ Household wealth index } \\
\hline Poorest & 192 & 88.7 & & \\
\hline $2^{\text {nd }}$ quintile & 161 & 85.2 & $0.6(0.3-1.2)$ & $0.8(0.4-1.5)$ \\
\hline $3^{\text {rd }}$ quintile & 139 & 72.0 & $0.3(0.2-0.5)^{* * * *}$ & $0.4(0.2-0.8)^{*}$ \\
\hline $4^{\text {th }}$ quintile & 137 & 62.4 & $0.2(0.1-0.4)^{* * * *}$ & $0.4(0.2-0.8)^{* *}$ \\
\hline Wealthiest & 96 & 55.4 & $0.1(0.1-0.3)^{* * *}$ & $0.3(0.1-0.8)^{*}$ \\
\hline \multicolumn{5}{|l|}{ Residence } \\
\hline Rural & 566 & 79.3 & & \\
\hline Urban & 160 & 61.6 & $0.4(0.3-0.6)^{* * *}$ & $0.7(0.5-1.4)$ \\
\hline \multicolumn{5}{|l|}{ Region } \\
\hline North & 250 & 79.5 & & \\
\hline Central & 324 & 70.3 & $0.6(0.4-0.9)^{*}$ & $0.8(0.5-1.2)$ \\
\hline South & 152 & 79.3 & $1.0(0.6-1.7)$ & $1.1(0.6-2.1)$ \\
\hline Total & 725 & 75.4 & & \\
\hline
\end{tabular}

${ }^{\mathrm{a}}$ Child born within two years preceding the survey; ${ }^{\mathrm{b}^{*}} \mathrm{p}<0.05,{ }^{* *} p<0.01,{ }^{* * *} p<0.001$.

educational status and from lower wealth quintiles also were more likely to continue to breastfeed their children at one year of age.

\section{Discussion and Program Implications}

In Lao PDR, breastfeeding remains the social norm with 95.6\% of children ever breastfed; and the prevalence of appropriate breastfeeding practices, including both exclusive and early initiation of breastfeeding practices have shown improvement. In the five year period between 2006 and 2011, there has been an increase from
$26 \%$ to $40 \%$ in exclusive breastfeeding and from $30 \%$ to $39 \%$ in early initiation of breastfeeding [10]. While this trend is positive, the use of infant formula has more than tripled during the same time period. This is particularly worrisome given that formula-fed infants are almost twice as likely to develop GI illness than children who are exclusively breastfeeding [11,12], Continued breastfeeding at both one year and two years of age has declined in the period between 2006 and 2011. In 2006, 82\% of children were still breastfeeding at one year of age [13], while this number had fallen to 73\% by 2011. 
In comparison with other ASEAN countries, the prevalence of early initiation of breastfeeding in Laos (39.6\%) was higher than national rates in Indonesia (29\%) and about the same as Vietnam (40\%), but lower than in the Thailand (50\%), Philippines (54\%), Cambodia (65\%) and Myanmar (76\%) [14]. In contrast, the prevalence of exclusive breastfeeding is higher than several ASEAN countries, including Thailand, Vietnam and Myanmar, although it is lower than Indonesia, Philippines and Cambodia. Finally, the prevalence of continued breastfeeding at one year of age in Laos (73\%) was lower than all other ASEAN countries except for Thailand, where just over half of all mothers are still breastfeeding when their infants are one year of age.

The analysis of key determinants of optimal breastfeeding practices revealed some important patterns which have implications for programming. After adjusting for wealth the factors of ethnicity, religion, and region of residence were found to have significant associations with breastfeeding patterns. Hence, major factors associated with exclusive breastfeeding and avoiding prelacteal feeds in Laos included minority non-Laotian ethnicity, non-Buddhist religion, and residence in the north. For these groups, the majority do not receive a prelacteal feed and are exclusively breastfed; this is not the case for ethnic Lao. The major difference between these groups and the ethnic Lao with respect to exclusive breastfeeding is the higher prevalence of formula feeding among ethnic Lao infants (13.7\% ethnic Lao infants under 6 months of age fed formula versus $2.5 \%$ - 5.4\% among other ethnic minority groups), the early feeding of complementary foods among ethnic Lao infants (27.3\% ethnic Lao infants under 6 months of age fed solid or semi-solid foods versus $6.7 \%-8.2 \%$ for Khmer and Hmong ethnic infants), and the higher use of water among ethnic Lao infants. These are unique features and require a keen understanding of the underlying social customs and norms in these settings to best design programs that will target barriers to improvement and that will appeal to the values of mothers toward postpartum practices and child care, which are invariably shaped by generations of tradition.

After adjusting for covariates in this study, while wealth status was not a major factor for early initiation of breastmilk, prelacteal feeding, and exclusive breastfeeding; there was a significant association between wealth and continued breastfeeding. The decreasing prevalence of continued breastfeeding since year 2006 is likely a result of decreases among urban, wealthy families. For exclusive breastfeeding the lack of a significant association with wealth and urban residence does not mean that these factors should be ignored. Formula use is more than 3 times higher in urban areas when compared to rural areas (40\% of children ages $0-23$ months fed for- mula in urban areas compared to $10.6 \%$ in rural); breastmilk substitutes are a major barrier to exclusive breastfeeding in urban areas and among wealthier households.

The public health sector is having a positive influence on early initiation of breastfeeding, but it is not yet a social norm for any group. Early initiation of breastfeeding was associated with having received antenatal care, delivery with the assistance of a skilled attendant, delivery at a public or private facility as compared to home deliveries, and higher maternal education. This suggests that the quality of training provided to the cadre of workers within the health care system is important in conveying IYCF messages and the influence of these workers is critical in the decision making by mothers in adopting the practice of early breastfeeding initiation. One of the barriers to early breastfeeding initiation can be prelacteal feeding, a practice that can also introduce harmful pathogens.

Receipt of antenatal care did not significantly alter rates of prelacteal feeds. However, when a traditional birth attendant (TBA) was present at birth or when a child was born in a private health facility, the likelihood of prelacteal feeding was significantly higher. These differences in prelacteal feeding practices highlight the strong influence of appropriate infant feeding counseling at time of delivery. Consequently, targeting TBAs with IYCF information and regulating the private sector to discourage promotion of breastmilk substitutes have the potential to greatly improve prelacteal feeding practices. Strategies are needed to include TBA's in communication efforts on early initiation and prelacteal feeding. For some ethnic minorities prelacteal feeding is not the social norm. For these groups more information is needed to determine why early initiation is not commonly practiced.

These findings provide an initial impression of the factors associated with optimal infant and feeding in Lao PDR, and highlight the complexity of designing effective programs. As the country embarks on an ambitious national plan to reduce the burden of chronic undernutrition, having a firm grasp of how to best influence feeding practices will be critical. The improvement of maternal, infant and early child nutrition requires a combination of interventions and strategic approaches, which will need to be designed and implemented in accordance with the unique social and cultural characteristics of the population in mind. The focus of such analyses need to consider the practical aspects of program design and implementation, and need to identify those population characteristics which may enable more effective targeting and crafting of communication messages. Many programs in the past have aimed to improve the "knowledge" of mothers regarding IYCF, but have not fully appreciated the importance of social norms and community dynamics. 
Consequently, community-based promotion and support need to be integral components of any IYCF program, which integrate an understanding of social norms as part of any health workers' training on infant feeding support and counseling.

\section{REFERENCES}

[1] J. Bryce, D. Coitinho, I. Darnton-Hill, et al., for the Maternal and Child Undernutrition Study Group, "Maternal and Child Undernutrition: Effective Action at National Level,” Lancet, Vol. 371, No. 9611, 2008, pp. 510-526. http://dx.doi.org/10.1016/S0140-6736(07)61694-8

[2] S. Horton, H. Alderman and J. A. Rivera, "The Challenge of Hunger and Malnutrition. Copenhagen Consensus Challenge Paper 2008,” In: BjørnLomborg, Ed., Global Crises, Global Solutions, 2nd Edition, Cambridge University Press, Cambridge, 2009.

[3] R. Feachem and M. Koblinsky, "Interventions for the Control of Diarrhoeal Diseases among Young Children," Bulletin of the WHO, Vol. 62, No. 2, 1984, pp. 271-291.

[4] C. Victoria, et al., "Evidence for Protection by BreastFeeding against Infant Deaths from Infectious Diseases in Brazil,” Lancet, Vol. 8, No. 8554, 1987, pp. 319-322. http://dx.doi.org/10.1016/S0140-6736(87)90902-0

[5] S. Arifeen, R. E. Black, G. Antelman, A. Baqui, L. Caulfield and S. Becker, "Exclusive Breastfeeding Reduces Acute Respiratory Infection and Diarrhea Deaths among Infants in Dhaka Slums,” Pediatrics, Vol. 108, No. 4, 2001, pp. 67-71. http://dx.doi.org/10.1542/peds.108.4.e67

[6] C. J. Chantry, C. R. Howard and P. Auinger, "Full Breast-
Feeding Duration and Associated Decrease in Respiratory Tract Infection in US Children,” Pediatrics, Vol. 117, No. 2, 2006, pp. 425-432. http://dx.doi.org/10.1542/peds.2004-2283

[7] WHO, "Infant and Young Child Feeding Counseling : An Integrated Course," World Health Organization, Geneva, 2006.

[8] M. J. Dibley, et al., “Across-Country Comparisons of Selected Infant and Young Child Feeding Indicators and Associated Factors in Four South Asian Countries," Food \& Nutrition Bulletin, Vol. 31, No. 2, 2010, pp. 366-375.

[9] N. Mangasarayan, et al., "Breastfeeding Promotion, Support and Protection: Review of Six Country Programmes," Nutrients, Vol. 4, No. 8, 2012, pp. 990-1014. http://dx.doi.org/10.3390/nu4080990

[10] Ministry of Health and Lao Statistics Bureau, "Lao Social Indicator Survey 2011-12, Final Report,” Ministry of Health and Lao Statistics Bureau, Vientiane, 2012.

[11] P. F. Chien and P. W. Howie, "Breast Milk and the Risk of Opportunistic Infection in Infancy in Industrialized and Non-Industrialized Settings,” Advances in Food \& Nutrition Research, Vol. 10, 2001, pp. 69-104.

[12] M. S. Kramer, B. Chalmers, E. D. Hodnett, et al., "Promotion of Breastfeeding Intervention Trial (PROBIT): A Randomized Trial in the Republic of Belarus," Journal of the American Medical Association, Vol. 285, No. 4, 2001, pp. 413-420. http://dx.doi.org/10.1001/jama.285.4.413

[13] "Demographic and Health Survey in Lao People's Democratic Republic,” 2006.

[14] Data from Child Info Data Base, 2013. http://www.childinfo.org/index.html 